Service social

\title{
Comportements suicidaires d'un jeune ou refus de recevoir des soins en santé mentale : quand la situation est signalée à la DPJ...
}

\author{
Danielle Nadeau, Denis Lacerte, Julie Tremblay-Roy et Myriam Sylvain
}

Volume 60, numéro 2, 2014

URI : https://id.erudit.org/iderudit/1027987ar

DOI : https://doi.org/10.7202/1027987ar

Aller au sommaire du numéro

Éditeur(s)

École de service social de l’Université Laval

ISSN

1708-1734 (numérique)

Découvrir la revue

Citer cet article

Nadeau, D., Lacerte, D., Tremblay-Roy, J. \& Sylvain, M. (2014). Comportements suicidaires d'un jeune ou refus de recevoir des soins en santé mentale : quand la situation est signalée à la DPJ.... Service social, 60(2), 1-13.

https://doi.org/10.7202/1027987ar
Résumé de l'article

Cette étude explore à partir d'une cohorte de jeunes en protection de la jeunesse $(\mathrm{N}=40079)$ des éléments non encore documentés dans la littérature. Elle examine la trajectoire de services de jeunes qui sont signalés aux autorités ou évalués pour des comportements suicidaires ou qui refusent des soins en santé mentale. La gestion du risque associé à ces jeunes diffère de ce qu'elle doit être pour les autres jeunes Québécois signalés aux autorités pour maltraitance : l'analyse de la compromission de leur sécurité semble notamment plus prudente. Cet article vise à éclairer les décideurs et concepteurs de programmes sur les enjeux associés à l'utilisation des services par les jeunes vulnérables, pour ainsi leur offrir des services toujours mieux adaptés et maximiser leur chance d'inclusion sociale. 


\title{
Comportements suicidaires d'un jeune ou refus de recevoir des soins en santé mentale : quand la situation est signalée à la DPJ...
}

\author{
NADEAU, Danielle \\ LACERTE, Denis \\ TREMBLAY-ROY, Julie \\ SYLVAIN, Myriam
}

\section{RÉSUMÉ}

Cette étude explore à partir d'une cohorte de jeunes en protection de la jeunesse ( $\mathrm{N}=40079)$ des éléments non encore documentés dans la littérature. Elle examine la trajectoire de services de jeunes qui sont signalés aux autorités ou évalués pour des comportements suicidaires ou qui refusent des soins en santé mentale. La gestion du risque associé à ces jeunes diffère de ce qu'elle doit être pour les autres jeunes Québécois signalés aux autorités pour maltraitance: l'analyse de la compromission de leur sécurité semble notamment plus prudente. Cet article vise à éclairer les décideurs et concepteurs de programmes sur les enjeux associés à l'utilisation des services par les jeunes vulnérables, pour ainsi leur offrir des services toujours mieux adaptés et maximiser leur chance d'inclusion sociale.

Mots clés: Comportements suicidaires, santé mentale, refus de soins, maltraitance, protection de la jeunesse

\section{ABSTRACT}

This study explores some poorly documented elements in child welfare services, by using administrative data $(\mathrm{N}=40,079)$. It looks at the service trajectory of youths reported to child welfare authorities or evaluated for suicidal ideation or acting out, or because of their refusal to receive mental health care threatens their security. The risk management of those youth differs from what it is for other youth signaled to the authorities for other abuse motives: It seems more cautious. This article aims to inform researchers and program designers about issues associated with the reality of those vulnerable youth in child welfare services, for then offering them more specialized services and maximise their social inclusion chance.

Keywords: Suicidal ideation, suicidal acting out, mental health, child abuse 


\section{INTRODUCTION}

Entre 2004 et 2007, environ 22,4\% de l'ensemble des décès survenus chez les Québécois âgés de 15 à 19 ans étaient causés par un suicide, plaçant le suicide au deuxième rang des causes de mortalité pour ce groupe d'âge (Gagné et Saint-Laurent, 2007). En 2005, on lui attribuait le tiers des décès des 15-19 ans (St-Laurent et Gagné, 2007). Pour les jeunes recevant des services des centres jeunesse, le taux de suicide a déjà été estimé jusqu'à cinq fois et demie supérieur à celui des autres Québécois du même âge (Farand et al., 2004).

Derrière la réalité douloureuse du suicide et des comportements suicidaires se trame souvent celle de la détresse psychologique et des maladies mentales ${ }^{1}$ qui l'accompagnent. À cet égard, les jeunes suivis par les services de protection de l'enfance présentent davantage de psychopathologies que ceux de la population générale. Selon les auteurs qui ont documenté la question, ces jeunes sont principalement affectés de troubles associés à des comportements dits « perturbateurs » (Garland et al., 2001 ; Hotte et al., 1996 ; Pauzé et al., 2004), et seraient portés à ressentir davantage de sentiments dépressifs et anxieux (De Bellis et al., 2001 ; Éthier, Lemelin et Lacharité, 2004) ou à répondre aux critères diagnostiques d'un trouble réactionnel de l'attachement (Nadeau et al., 2010 ; Zeanah et al., 2004 ; Zeanah, Smyke et Dumitrescu, 2002 ; voir Nadeau et al., 2012, pour une recension de la littérature). De plus, ces jeunes cumulent un ensemble de facteurs de risques associés au fait de vivre en contexte de grande adversité et au stress chronique qui l'accompagne comme la pauvreté, l'exposition à diverses formes de violence, les difficultés personnelles et scolaires, ou l'exclusion sociale de leurs parents, etc. (Cloutier et al., 2008 ; Kerker et Doore, 2006 ; Morin et al., 2012).

Pour maximiser leurs chances d'inclusion sociale en leur offrant des services toujours mieux adaptés, il s'avère important d'éclairer les décideurs et concepteurs de programmes sur des enjeux associés à l'utilisation des services requis par cette clientèle très vulnérable au plan de sa santé mentale (Rodriguez et al., 2006 ; Romanelli et al., 2009). Nous étendons cette perspective développée en santé mentale à l'utilisation des services sociaux spécialisés requis en protection de la jeunesse par cette clientèle. Nous en savons encore très peu, en effet, sur le vécu des jeunes suicidaires ou affectés de troubles mentaux, diagnostiqués et non soignés, lorsque leur situation est signalée à la Direction de la protection de la jeunesse. Dans quelle proportion ces jeunes affectés sont-ils présents parmi les signalements? Quelles sont leurs caractéristiques personnelles (c'est-à-dire âge et sexe) et en quoi leur parcours au sein des services de protection offerts est-il différent de celui des jeunes signalés aux autorités pour d'autres motifs ? Par ailleurs, nous en savons encore moins sur la réalité des jeunes de moins de 12 ans qui peuvent, eux aussi, souffrir en silence de détresse psychologique. Ils constituent une clientèle encore plus vulnérable au plan de la santé mentale du fait de leur jeune âge et ils

\footnotetext{
1 La perspective proposée par l'Organisation mondiale de la santé (2001) et situant la santé mentale dans un contexte de "santé globale » est retenue. Ainsi la "maladie mentale » (ou trouble mental) correspond à des " affections cliniquement significatives qui se caractérisent par un changement du mode de pensée, de l'humeur (affects) ou du comportement associé à une détresse psychique et/ou à une altération des fonctions mentales ». Elle ne constitue pas de simples variations du comportement. Pour être considérées comme des maladies mentales, les " anomalies doivent être permanentes et répétées, et causer une souffrance ou constituer un handicap dans un ou plusieurs domaines de la vie courante »(OMS, 2001, p. 21).
} 
ont, jusqu'à ce jour, été très peu étudiés dans la littérature. Ce sont ces questions de recherche qui ont motivé la réalisation de notre étude.

\section{OBJECTIFS}

Cette recherche poursuit l'objectif de fournir des données originales concernant le portrait et la trajectoire de services en protection de la jeunesse (PJ), de jeunes nouvellement signalés aux autorités lorsque des comportements suicidaires ${ }^{2}$ ou des problèmes de santé mentale connus et non traités menacent directement leur sécurité ou leur développement. Ces éléments sont étudiés en explorant les bases de données administratives anonymisées des établissements. De façon plus spécifique, nous allons:

1) documenter, au sein d'une cohorte générale de nouveaux signalements en $\mathrm{PJ}^{3}$, le taux de prévalence des jeunes signalés en raison d'un comportement ou d'une idéation suicidaire, ou parce que des soins en santé mentale requis par leur état sont non assurés ou refusés (le " groupe cible »);

2) documenter le pourcentage de rétention des signalements, le taux de judiciarisation des situations et le parcours du groupe cible dans chacune des étapes du processus PJ, soit le signalement, l'évaluation et l'orientation, en les comparant à ceux de l'ensemble des autres signalements reçus (le « groupe général »);

3) vérifier s'il y a des différences entre le groupe cible et le groupe général selon le sexe, l'âge ou les motifs juridiques de signalement et de compromission ;

4) documenter ces mêmes variables auprès d'une sous-cohorte de jeunes âgés de moins de 12 ans.

\section{MÉTHODOLOGIE}

Cette étude de cohorte, quantitative et exploratoire, privilégie un devis descriptif mettant en valeur l'existence d'associations ou de différences.

\section{Procédure}

Une demande d'extraction de données des dossiers informatisés des usagers recevant des services en protection de la jeunesse, au cours de la période comprise entre le $1^{\mathrm{er}}$ septembre 2007 et le $1^{\mathrm{er}}$ avril $2010^{4}$ (15 mois), a été acheminée à tous les centres jeunesse participants. En effet, la cohorte à l'étude a été extraite de l'ensemble des signalements reçus entre le $1^{\mathrm{er}}$ septembre 2007 et le $1^{\mathrm{er}}$ septembre 2008. Néanmoins, les données présentées dans cet article constituent des données partielles d'un projet longitudinal plus large qui visait à étudier la trajectoire des jeunes sur une période de 15 mois suivant leur signalement, justifiant ainsi la demande d'extraction des données jusqu'en avril 2010.

\footnotetext{
${ }^{2}$ Les « comportements suicidaires ", comme on les entend ici, comprennent les tentatives ou idéations suicidaires sérieuses, susceptibles de constituer une menace pour la sécurité du jeune (p. ex. idéation avec un plan défini).

${ }^{3}$ C'est-à-dire sans suivi actif dans un centre jeunesse au moment où leur situation est rapportée aux autorités.

${ }^{4}$ Cette période de 15 mois à l'application des mesures (AM) constituait le maximum disponible lorsque nous avons débuté l'étude.
} 
Une entente de transfert et d'utilisation des données a été signée avec chacun des centres jeunesse participants et les formalités éthiques pour une recherche multicentrique ont été complétées. Ces centres ont fait parvenir à l'équipe de recherche, sur un CD crypté, les différentes tables de données nécessaires. Un membre de l'équipe de recherche a pris contact avec la personne-ressource du domaine informationnel du centre jeunesse expéditeur, afin de procéder au décryptage du CD. Après la réception de toutes les bases locales (en format ACCES), chacune de celle-ci a fait l'objet d'une première inspection de contenu. Les dates correspondant à la période couverte par l'étude ont été vérifiées. Par la suite, les données ont été agglomérées en une seule base sur Oracle. Une première série de requêtes en $S Q L$ (Structured Query Language) a été appliquée sur ces données brutes afin de créer des fichiers à l'aide du logiciel Statistical Package for the Social Sciences (SPSS, version 13). Des examens de contenu pour s'assurer de la cohérence des données et la recherche de doublons ont par la suite été effectués sur ces fichiers ${ }^{5}$. Finalement, les requêtes SQL ont été raffinées et appliquées aux données brutes une nouvelle fois afin de corriger les erreurs observées et créer des fichiers de recherches finaux à l'aide du programme SPSS.

\section{Établissement des cohortes étudiées}

Sur les 16 centres jeunesse contactés, 13 ont accepté de participer à l'étude. La cohorte à l'étude comprend l'ensemble des signalements reçus entre le $1^{\text {er }}$ septembre 2007 et le $1^{\text {er }}$ septembre 2008 par les centres jeunesses participants. Ces signalements ( $\left.N=48559\right)$ concernent des jeunes âgés de 0 à 17 ans recevant des services en vertu de la Loi sur la protection de la jeunesse (LPJ). Certains jeunes ayant été signalés plus d'une fois, la cohorte totale de l'étude est de quarante mille soixante-dix-neuf jeunes ( $N=40079)$. Le groupe cible est composé des jeunes qui, parmi cette cohorte, font l'objet de l'un des motifs de rétention ou de compromission suivant :

a) des soins de santé mentale non assurés ou refusés par le ou les parent(s) ou le milieu substitut ; ou

b) un refus du jeune de recevoir des soins pour sa santé mentale ; ou

c) un comportement suicidaire (idéation divulguée, verbalisation ou acte physique).

À cet effet, il est à noter que le groupe cible est constitué de jeunes qui ont été signalés aux autorités pour l'un de ces motifs, mais aussi de jeunes qui ont été identifiés pour des comportements suicidaires ou refus de soin en cours de trajectoire, soit par exemple après évaluation ou orientation. Le groupe de comparaison général est quant à lui constitué des autres jeunes qui ne répondent pas à ces critères.

\section{ANALYSES}

Dans un premier temps, des analyses ont été effectuées afin de documenter la prévalence des signalements concernant des jeunes du groupe cible (objectif 1). Des taux d'incidence sont calculés à chacune des différentes étapes du processus en PJ, c'est-à-dire du signalement à

5 Exemple de vérification : dans une cohorte de nouveaux signalements retenus (et non de dossiers actifs), il doit y avoir au moins une problématique principale par signalement si on décide de retenir le cas pour évaluation. 
l'application des mesures de protection (objectif 2). Ce processus est déterminé par le cadre juridique québécois en protection de l'enfance, statuant sur toutes les étapes à suivre ainsi que sur les conditions donnant ouverture à l'intervention (Loi sur la Protection de la jeunesse, c. P 34.1, L.R.Q.) ${ }^{6}$. Lors de l'évaluation de la situation par les autorités, nous avons calculé trois taux : celui des situations où la sécurité et le développement sont compromis (SDC); celui des situations où les faits sont fondés ou non, mais la sécurité et le développement sont non compromis (FFSDNC + FNFSDNC) ; et, finalement, un taux calculé pour l'ensemble des autres situations (AUTRES) ${ }^{7}$. Lors des étapes cliniques nécessaires pour déterminer la prise en charge et la nature du suivi psychosocial (étape dite de l'orientation), trois autres taux sont calculés : un taux pour les fermetures de dossier; un pour les mesures volontaires; et un pour les mesures judiciaires. Finalement, des tableaux croisés (khi carré) entre le groupe cible et le groupe général sur les variables nominales suivantes sont effectuées afin de vérifier la présence de relations et, les cas échéants, la force de cette association (objectif 3 ) : le genre, les motifs juridiques de compromission (LPJ) et les personnes responsables du signalement. Des analyses complémentaires sont effectuées à titre exploratoire (objectif 4).

\section{RÉSULTATS}

\section{Caractéristiques socio démographiques}

Le groupe cible est constitué à 51,6\% $(n=980)$ de garçons et à 48,4 \% $(n=873)$ de filles. La répartition du genre est pratiquement identique dans le groupe de comparaison général, avec $51,1 \%(n=19569)$ de garçons et 48,6\% $(n=18614)$ de filles. Les jeunes du groupe cible sont plus âgés : l'âge moyen lors du premier signalement est de 13,9 ans ( $E T=3,3$ ans). II est de 9,5 ans $\left(E T=5,1\right.$ ans) pour les autres jeunes signalés aux autorités ${ }^{8}$.

\section{Trajectoire de services}

L'étude documente l'utilisation de certains services en PJ de la clientèle cible en comparaison avec les autres jeunes, du signalement à l'application des mesures de protection. Les jeunes du groupe cible sont signalés plus fréquemment par des professionnels $(77,3 \%)$ que ceux du groupe général $(67,4 \%)$ et cette différence est significative $[X(3, N=48599)=110, p<0,01]$. La figure 1 rapporte les différents taux de prévalence et d'incidence calculés pour la trajectoire des signalements entre ces deux groupes de jeunes, à chacune de ces étapes.

6 L'incidence des signalements retenus se calcule en divisant le nombre de signalements retenus pour le groupe cible de base par le nombre total de signalements retenus, le tout multiplié par 100. La méthode de calcul est la même pour l'ensemble du processus PJ (ou trajectoire PJ).

7 Cette catégorie comprend les situations où il y a une incapacité de procéder à l'évaluation, un décès du jeune, un transfert à un autre $\mathrm{CJ}$ et des décisions non rendues.

8 Rappelons qu'il y a un nombre moins élevé de jeunes que de signalements. De plus dans le groupe général, il y a 93 signalements où le sexe est « inconnu ». Ces situations correspondent à des signalements non retenus où des informations de base n'ont pas été saisies dans le dossier informatisé de l'usager par le clinicien. 


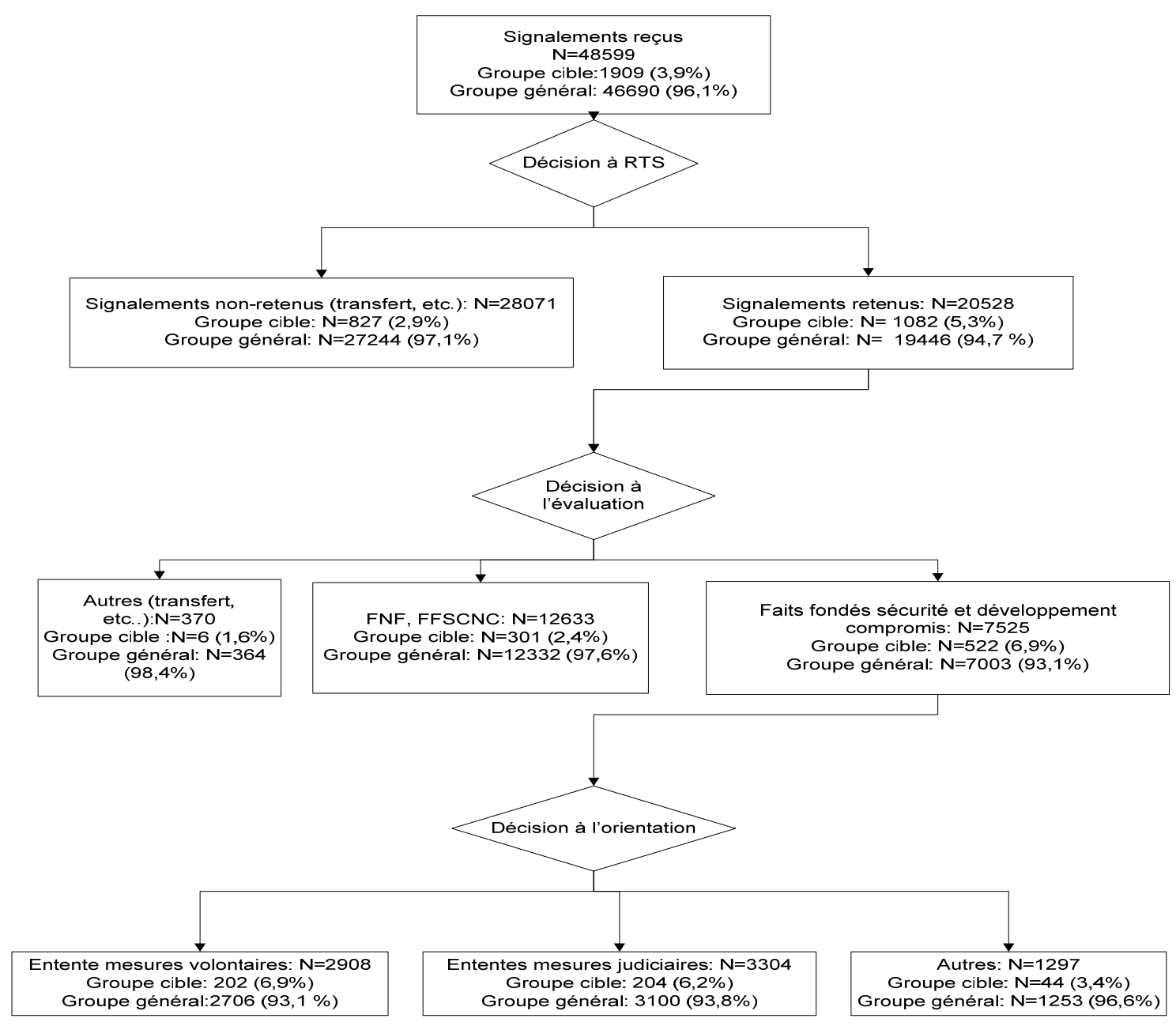

Figure 1 : Trajectoire de services et taux de prévalence et d'incidence

Le taux de rétention de l'ensemble des signalements du groupe cible est significativement plus élevé $(56,7 \%)$ que celui du groupe général $(41,6 \%)\left[\chi_{2}(1, N=48599)=169,8, p<0.01\right]$. De plus, la proportion de signalements du groupe cible passe de 3,9\% (1909 / 48 599) lors de la réception de la demande de signalement, à $5,3 \%$ au moment de la rétention de celui-ci (1082 / 20 528). La gestion du risque de compromission distingue les deux groupes dès le moment de la décision de retenir les signalements pour procéder à une évaluation plus approfondie.

Après cette évaluation, les situations où la sécurité et le développement sont considérés compromis (FFSDC) est de $63 \%$ au sein du groupe cible, comparativement à $35,5 \%$ pour le groupe général. Cette différence est significative $\left[X_{2}(3, N=20528)=288,5, p<0.01\right]$. Près des deux tiers des évaluations du risque psychosocial pour déterminer si la sécurité du jeune est compromise concluent que c'est effectivement le cas. Parmi ces 552 situations, 369 d'entre elles $(70,7 \%)$ étaient déjà incluses dans le groupe cible à l'étape du signalement. Les difficultés présentes au moment du signalement persistent et sont confirmées pour une très grande proportion des jeunes du groupe cible, d'une étape à l'autre. De plus, suite à l'évaluation psychosociale approfondie des jeunes, la véracité des faits rapportés est très souvent 
confirmée : dans $92,6 \%$ des situations du groupe cible, les faits sont estimés fondés, alors que la proportion est de $73,9 \%$ pour le groupe général.

\section{Motifs de compromission}

Les motifs des signalements reçus sont présentés au sein du tableau 1.

Tableau 1

Motifs à l'appui des signalements reçus

\begin{tabular}{lccc}
\hline \multirow{2}{*}{ Motif principal de signalement } & \multicolumn{2}{c}{ Groupe } & \\
\cline { 2 - 3 } & $\begin{array}{c}\text { Cible } \\
(\mathbf{n}, \%)\end{array}$ & $\begin{array}{c}\text { Général } \\
(\mathbf{n}, \%)\end{array}$ & $\begin{array}{c}\text { Total } \\
(\mathbf{n}, \%)\end{array}$ \\
Négligence & $418(21,9 \%)$ & $13996(35,1 \%)$ & $14414(34,5 \%)$ \\
Abus physique & $78(4,1 \%)$ & $7121(17,8 \%)$ & $7199(17,2 \%)$ \\
Abus sexuel & $30(1,6 \%)$ & $4470(11,2 \%)$ & $4500(10,8 \%)$ \\
Troubles de comportement & $1303(68,3 \%)$ & $8243(20,6 \%)$ & $9546(22,8 \%)$ \\
Abandon & $7(0,4 \%)$ & $211(0,5 \%)$ & $218(0,5 \%)$ \\
Mauvais traitement psychologique & $73(3,8 \%)$ & $5888(14,7 \%)$ & $5961(14,2 \%)$ \\
\hline Total & $1909(100 \%)$ & $39929(100 \%)$ & $41838(100 \%)$ \\
\hline
\end{tabular}

\footnotetext{
${ }^{a}$ Le nombre total de "problématiques » invoquées (ou motifs juridiques de rétention ; $n=41$ 838) est plus petit que le nombre total de signalements $(n=48599)$ car pour les cas non retenus, cette information n'est pas obligatoire et souvent, peu disponible. Les 6295 problématiques manquantes sont associées au groupe général.
}

Davantage de jeunes affichent des troubles de comportement sérieux dans le groupe cible $\left[X_{2}(5, N=41838)=2412, p<0.05\right]$. Les motifs juridiques concernant les signalements retenus sont présentés pour leur part dans le tableau 2 . 
Tableau 2

Motifs juridiques à l'appui des signalements retenus

\begin{tabular}{lccc}
\hline \multirow{2}{*}{ Problématique principale } & \multicolumn{3}{c}{ Groupe } \\
\cline { 2 - 4 } & $\begin{array}{c}\text { Cible } \\
(\mathbf{n}, \%)\end{array}$ & $\begin{array}{c}\text { Général } \\
(\mathbf{n}, \%)\end{array}$ & $\begin{array}{c}\text { Total } \\
\mathbf{( n ,} \%)\end{array}$ \\
Négligence & $271(25,0 \%)$ & $6973(35,9 \%)$ & $7244(35,3 \%)$ \\
Abus physique & $61(5,6 \%)$ & $5221(26,8 \%)$ & $5282(25,7 \%)$ \\
Abus sexuel & $27(2,5 \%)$ & $2235(11,5 \%)$ & $2262(11,0 \%)$ \\
Troubles de comportement & $666(61,6 \%)$ & $2037(10,5 \%)$ & $2703(13,2 \%)$ \\
Abandon & $6(0,6 \%)$ & $141(0,7 \%)$ & $147(0,7 \%)$ \\
Mauvais traitement psychologique & $51(4,7 \%)$ & $2839(14,6 \%)$ & $2890(14,1 \%)$ \\
\hline Total & $1082(100 \%)$ & $19446(100 \%)$ & $20528(100 \%)$ \\
\hline
\end{tabular}

Encore à cette étape dans la trajectoire de services, davantage de jeunes du groupe cible devront se prêter à une évaluation subséquente de leurs troubles de comportements. La négligence, l'abus physique, l'abus sexuel et les mauvais traitements psychologiques sont plus élevés dans le groupe général $\left[X_{2}(5, N=20528)=2890,8, p<0.01\right]$. Par ailleurs, la proportion de jeunes du groupe cible dont la rétention du signalement a trait à un comportement suicidaire est ici de $60,4 \%(n=638)$.

\section{Évaluation}

Une fois qu'il a été statué que la sécurité du jeune est compromise, le jeune bénéficiera d'une évaluation psychosociale approfondie de sa situation. À cette étape, la proportion de signalements se référant à des troubles de comportements, au sens de l'article $38 \mathrm{~d}$, s'avère toujours beaucoup plus élevée pour les jeunes du groupe cible $(60,6 \%)$ que pour ceux du groupe général $(16,1 \%)$. La différence est significative $\left[X_{2}(5, N=7525)=635,8, p<0.01\right]$. Rappelons que les jeunes du groupe cible sont également plus vieux.

\section{Orientation}

Suite à l'évaluation psychosociale approfondie de la situation, quand la sécurité et le développement d'un jeune sont toujours considérés compromis, le travailleur social ${ }^{9}$ (au nom du directeur de la protection de la jeunesse) complète son analyse de façon à déterminer l'orientation du dossier pour la prise en charge. C'est ici que des ententes sont prises avec les parents et le jeune pour corriger la situation. Lorsque la décision est contestée par les parents ou le jeune, l'orientation peut déboucher sur des mesures dites «judiciaires». Le tableau 3 présente les résultats pour l'orientation des situations examinées dans notre étude.

9 Ou la travailleuse sociale 
Tableau 3

Orientation des dossiers en fonction des groupes

\begin{tabular}{lccc}
\hline & \multicolumn{2}{c}{ Groupe } \\
\cline { 2 - 4 } Décision à l'orientation & $\begin{array}{c}\text { Cible } \\
(\mathbf{n}, \%)\end{array}$ & $\begin{array}{c}\text { Général } \\
(\mathbf{n}, \%)\end{array}$ & $\begin{array}{c}\text { Total } \\
(\mathbf{n}, \%)\end{array}$ \\
Entente sur mesures volontaires & $199(44,2 \%)$ & $2656(37,5 \%)$ & $2855(37,9 \%)$ \\
Application des mesures judiciaires & $200(44,4 \%)$ & $3019(42,7 \%)$ & $3219(42,8 \%)$ \\
Autres $^{a}$ & $51(11,3 \%)$ & $1400(19,8 \%)$ & $1451(19,3 \%)$ \\
\hline Total $^{2}$ & $450(100 \%)$ & $7075(100 \%)$ & $7525(100 \%)$ \\
\hline
\end{tabular}

a La catégorie "autres " à l'étape d'orientation comprend les décisions suivantes: transfert à un autre CJ, intervention terminale à terme, rejet de la requête par le tribunal, arrêt de l'orientation pour un autre motif et autre type de transfert.

Une proportion significativement plus élevée de mesures volontaires est présente dans le groupe cible $\left[X_{2}(2, N=7525)=20,9, p<0.01\right]$. Quant aux motifs justifiant l'intervention sociale en protection, on note encore une plus grande proportion de jeunes présentant des troubles de comportement au sens de l'article $38 \mathrm{~d}$ dans le groupe cible (68\% contre $19 \%$ pour le groupe général), et ce, pour tous les types de mesures, convenues ou imposées. La proportion des jeunes qui présentent uniquement un comportement suicidaire au moment de leur orientation vers un suivi social est de 46,9\% $(n=187)$. Celle des jeunes dont le refus de soins en santé mentale menace leur sécurité ou leur développement est de 45,4\% $(n=181)$. Cette dernière proportion est donc plus importante à cette étape de la trajectoire de services qu'au moment de la rétention des signalements. Une proportion plus faible de jeunes présente à la fois les deux difficultés, soit $7,7 \%(n=31)$. De plus, les jeunes qui sont orientés vers une prise en charge sociale liée à un refus de soins pour leur santé mentale sont plus jeunes $(\underline{M}=11,9$ ans ; $\underline{E T}=3,9)$ que ceux orientés en raison de comportements suicidaires ( $\underline{M}=14,9$ ans ; et $\underline{E T}=1,9)$.

\section{Analyses exploratoires : Les enfants de moins de 12 ans et la trajectoire PJ}

Près de 30000 signalements examinés au sein de la cohorte étudiée concernent des enfants de moins de 12 ans ( $n=28262)$, dont quatre cents jeunes $(n=400)$ assignés au groupe cible. Notre examen met en relief que $63 \%$ de ces signalements sont d'abord effectués en raison d'un refus de soins. Toutefois, $36 \%$ de ceux-ci ( $n=144$ jeunes) concernent des comportements suicidaires manifestés par l'enfant, et estimés susceptibles de menacer sa sécurité ou son développement. Le taux de rétention des signalements pour les jeunes du groupe cible de 12 ans et moins s'avère également assez élevé (60,6 \%). Après l'évaluation psychosociale, quatre-vingt-douze (92) de ces enfants plus jeunes du groupe cible sont orientés pour l'application de mesures particulières. La proportion de mesures volontairement consenties est équivalente à celles des mesures ordonnées par voie judiciaire au sein de ce sous-groupe d'enfants. Parmi eux, près de $82 \%(n=75)$ font l'objet d'un refus ou de soins non assumés par les parents. Ces enfants sont âgés en moyenne de 7,9 ans $(\underline{E T}=2,8)$, alors que les enfants signalés en raison d'idéations ou 
de comportements suicidaires $(n=17)$ sont significativement un peu plus vieux $(10,1$ ans; $\underline{E T}=1,2)(F(1,90)=10,14, p=0.002)$.

\section{DISCUSSION}

La principale limite de notre étude est qu'elle ne permet pas d'identifier et de documenter la situation de tous les jeunes suivis par les centres jeunesse qui présentent des idéations suicidaires ou une souffrance psychologique subjective importante. Toutefois, elle fournit des données empiriques originales au sujet des particularités concernant des jeunes dont la prise en charge sociale est justifiée en raison de leurs comportements suicidaires ou de leurs refus de recevoir des soins en santé mentale requis par leur état. Le faible taux des jeunes répondant aux critères du groupe cible démontre qu'en général, les jeunes Québécois sont signalés à la Direction de la protection de la jeunesse pour des motifs bien différents que les comportements suicidaires ou un refus de recevoir des soins. Cette donnée doit être interprétée avec une grande prudence : bien des jeunes sont possiblement suicidaires ou affectés d'une souffrance psychologique importante non détectée, si l'on se fie à la documentation clinique et scientifique concernant cette population clinique (Farand, et al., 2004 ; Garland et al., 2001 ; Pauzé et al., 2004 ; Nadeau et al., 2012). Les jeunes détectés plus vieux affichent une plus grande proportion de troubles de comportement sérieux et cette proportion plus importante ne surprend pas. Ces jeunes sont surtout dans un "agir » qu'ils retournent contre eux-mêmes. En ce qui les concerne, la gestion du risque s'effectue de façon assez serrée par les techniciens en travail social et les travailleurs sociaux exerçant en centres jeunesse. Les bénéfices associés aux efforts d'implantation des protocoles d'intervention en situation de problématique suicidaire (ACJQ, 2000) et des équipes de $2^{\mathrm{e}}$ niveau prévus au Plan d'action en santé mentale (20052010) au cours des dernières années semblent se traduire positivement sur le terrain. Les distinctions observées dans la gestion du risque pourraient être attribuables, en partie, à l'expertise développée par les cliniciens suite à l'implantation de ces mesures.

La proportion de jeunes du groupe cible dont la rétention du signalement a trait à un comportement suicidaire est beaucoup plus importante que celle des jeunes présentant un refus de soins pour un problème de santé mentale connu et non traité. Cependant, elle diminue de $20 \%$ au fur et à mesure que l'on avance dans la trajectoire de services en PJ, au profit des jeunes qui ont fait l'objet d'un refus de soins. II est possible qu'après l'évaluation ou même une fois que le signalement est retenu, les jeunes identifiés comme suicidaires reçoivent des services adéquats permettant, en partie, de faire en sorte que des soins leur soient prodigués et que leur sécurité ne soit plus menacée. En effet, une proportion significativement plus élevée de mesures volontaires était constatée dans le groupe cible. De même, des jeunes non détectés au plan de leur santé mentale au moment de la rétention du signalement, le sont possiblement par la suite grâce à l'évaluation de leur situation par un travailleur social. Celle-ci intervient plus loin dans la trajectoire en PJ. Les jeunes orientés pour une prise en charge sociale liée à un refus de soins sont plus jeunes d'au moins trois ans que ceux présentant des comportements suicidaires. II est possible qu'après l'intervention évaluative du travailleur social, le jeune ou le parent accepte une mesure volontaire permettant ici aussi de résorber la situation. Cette explication ne semble toutefois ne pas convenir aux enfants plus jeunes où la situation est inversée : leur 
proportion est plus importante au sein du groupe cible en raison d'un refus de soins et la proportion de mesures volontaires et judiciaires est équivalente dans leur cas.

En conclusion, malgré ses limites méthodologiques certaines, cette étude permet de jeter un regard critique sur l'importance de continuer à prioriser le dépistage de la souffrance psychologique chez les usagers des centres jeunesse. Dans une perspective de prévention, une attention particulière devrait être portée aux jeunes âgés de moins de 12 ans, même s'ils " dérangent » moins que les adolescents. Nous espérons que certains travaux actuellement en cours à l'ACJQ et au ministère de la Santé et des Services sociaux aident à pallier ces lacunes au plan de l'organisation des services. Au moment d'écrire ces lignes, des protocoles de dépistage sont actuellement en expérimentation et les protocoles actuels d'intervention en situation de problématique suicidaire sont en processus avancé de révision. Idéalement, leur implantation devrait se traduire par des adaptations du dossier informatisé des usagers pour éventuellement fournir aux chercheurs des données longitudinales permettant de dresser le portrait réel de l'évolution de la santé mentale des jeunes victimes de maltraitance. Des jeunes considérés parmi les plus vulnérables de notre société au plan de leur inclusion sociale. Davantage de travaux doivent continuer à approfondir en priorité cette réalité troublante afin de mieux guider les interventions.

NADEAU, Danielle Psychologue-chercheuse Centre jeunesse de Québec Institut universitaire

LACERTE, Denis Agent de planification, de programmation et de recherche Centre jeunesse de Québec Institut universitaire

TREMBLAY-Roy, Julie Étudiante à la maîtrise École de service social Université Laval

SYLVAIN, Myriam Étudiante en doctorat École de service social Université Laval

\section{BIBLIOGRAPHIE}

ACJQ (Association des centres jeunesse du Québec) (2000). Protocole d'intervention en situation de problématique suicidaire dans les centres jeunesse. Montréal, ACJQ. 
Cloutier, R., D. Nadeau, S. Bordeleau et M. Verreault (2008). "Pauvreté, santé mentale et protection de la jeunesse ». In A. Demers, M. De Konninck et K. Frohlich. (dir.), Les inégalités sociales de santé au Québec Montréal, Presses de l'Université de Montréal, p. 263-291.

De Bellis, M.D., E.R. Broussard, D.J. Herring, S. Wexler, G. Moritz et J.G. Benitez (2001). "Psychiatric co-morbidity in caregivers and children involved in maltreatment: a pilot research study with policy implications ». Child Abuse \& Neglect, vol. 25, nº 7, p. 923-944.

Éthier, L.S., J.P. Lemelin et C. Lacharité (2004). «A longitudinal study of the effects of chronic maltreatment on children's behavioral and emotional problems ». Child Abuse \& Neglect, vol. $28, n^{\circ} 12$, p. $1265-1278$.

Farand, L., F. Chagnon, J. Renaud et M. Rivard (2004). "Completed suicides among Quebec adolescents involved with juvenile justice and child welfare services ». Suicide Life Threat Behavior, vol. 34, n 1, p. 24-35.

Flynn, R.J. et T.D. Aubry (1999). «Integration of persons with developmental or psychiatric disabilities: Conceptualization and measurement ». In R.J. Flynn et R.A. Lemay (dir.), $A$ quarter-century of normalization and social role valorization: Evolution and impact. Ottawa, University of Ottawa Press, p. 271-303.

Gagné, M. et Saint-Laurent, D. (2007). La mortalité par suicide au Québec: tendances et données récentes - 1981 à 2007. Institut national de santé publique du Québec (INSP). Accessible en ligne <http://www.inspq.qc.ca/pdf/publications/890_BulSuicide09.pdf.

Garland, A.E., R.L. Hough, K.M. McCabe, M. Yeh, P.A. Wood et G.A. Aarons (2001). Prevalence of psychiatric disorders in youths across five sectors of care. Journal of the American Academy of Child and Adolescent Psychiatry, vol. 40, p. 409-418.

Hotte, J.-P., M. Déry, R. Pauzé et J. Toupin (1996). Étude des caractéristiques sociofamiliales et personnelles associées au placement d'enfants en centre de réadaptation pour jeunes en difficulté d'adaptation dans la région de Montréal. Montréal, Centre Jeunesse de Montréal.

Kerker, B.D. et M.M. Dore (2006). « Mental Health Needs and Treatment of Foster Youth: Barriers and Opportunities ». American Journal of Orthopsychiatry, vol. 76, $\mathrm{n}^{\circ}$ 1, p. 138-147.

Légaré, G., M. Gagné, M., D. St-Laurent et P.-A. Perron, (2013). La mortalité par suicide au Québec: 1981 à 2010. Mise à jour 2013. Québec, Institut national de santé publique du Québec. Accessible en ligne

<http://www.aqps.info/media/documents/Suicide_Qc_INSPQ_miseajour2013.pdf>.

Morin, J., M.-E. Nadeau, D. Nadeau, M. Bigras, M.-C. Guay et P. Nolin (2012). « Portrait du développement cognitif de l'enfant victime de maltraitance ». In M.-H. Gagné, S. Drapeau et M.-C. St-Jacques (dir.), Les enfants maltraités: de l'affliction à l'espoir. Pistes de compréhension et d'action. Québec, Presses de l'Université Laval, p. 93-119.

Nadeau, D., Bergeron-Leclerc, C., E. Pouliot, M. Chantal et M. Dufour, M. (2012). « Troubles mentaux chez les jeunes victimes de maltraitance ». In M.-H. Gagné, S. Drapeau et M.-C. StJacques (dir.), Les enfants maltraités: de l'affliction à l'espoir. Pistes de compréhension et d'action. Québec, Presses de l'Université Laval, p.71-85. 
Nadeau, D., F. Malingrey, M. Chantal et S. Bonneville (2010). La santé mentale des usagers du Centre Jeunesse de Québec - Institut universitaire: Suivi préliminaire d'implantation de l'Offre de services en santé mentale du CJQ-IU. Québec: Centre jeunesse de QuébecInstitut universitaire.

Nadeau, D., et M.-R. Patry (2008). La santé mentale des usagers du Centre jeunesse de Québec-Institut universitaire. Modèle de développement et d'organisation des services. Québec, Centre jeunesse de Québec - Institut universitaire.

OMS (Organisation mondiale de la santé) (2001). Rapport sur la santé dans le monde 2001. La santé mentale : nouvelle conception, nouveaux espoirs. Genève, Organisation mondiale de la santé. Accessible en ligne <http://www.who.int/whr/2001/en/whr01_fr.pdf?ua=1>.

Pauzé, R., J. Toupin, M. Déry, H. Mercier, M. Cyr, F. Cyr, J.-Y. Frappier, C. Chamberland et M. Robert (2004). Portrait des jeunes âgés de 0 à 17 ans référés à la prise en charge des centres jeunesse du Québec, leur parcours dans les services et leur évolution dans le temps. Sherbrooke, Université de Sherbrooke, Groupe de recherche sur les inadaptations psychosociales de l'enfance (GRISE).

Rodriguez, L., L. Bourgeois, Y. Landry, L. Guay et J.-L. Pinard (2006). Repenser la qualité des services en santé mentale dans la communauté. Changer de perspectives. Québec, Presses de l'Université du Québec.

Romanelli, L.H., K.E. Hoagwood, S.J. Kaplan, S.P. Kemp, R.L. Hartman, C. Trupin, W. Soto, P.J. Pecora, T.L. LaBarrie et P.S. Jensen (2009). « Best practices for mental health in child welfare : parent support and youth empowerment guidelines ». Child Welfare, vol. 88, $\mathrm{n}^{\circ} 1$, p. $189-212$.

St-Laurent, D., et M. Gagné (2007). Surveillance des suicides au Québec : les décès imputables aux suicides dans la population québécoise. Québec, Institut national de santé publique du Québec (INSPQ).

St-Laurent, D., et M. Gagné, (2008). Surveillance des suicides au Québec : ampleur et évolution du problème de 1981 à 2006. Québec, Institut national de santé publique du Québec (INSPQ). Accessible en ligne :

<http://www.inspq.qc.ca/pdf/publications/734_suicidequebec2008.pdf>.

Zeanah, C.H., M. Scheeringa, N.W. Boris, S.S. Heller, A.T. Smyke et J. Trapani (2004). " Reactive attachment disorder in maltreated toddlers ". Child Abuse \& Neglect, vol. 28, $\mathrm{n}^{\circ} 8$, p. 877-888.

Zeanah, C.H., A.T. Smyke et A. Dumitrescu (2002). "Attachment disturbances in young children. II. Indiscriminate behavior and institutional care ». Journal of the American Academy of Child \& Adolescent Psychiatry, vol. 41, nº 8, p. 983-989. 